\title{
SECOND ORDER PARALLEL TENSOR IN REAL AND COMPLEX SPACE FORMS
}

\author{
RAMESH SHARMA \\ Department, of Mathernatics \\ Michigan State University \\ kast Lansing, Michigan 48824, USA \\ (Received February 9, 1988)
}

\begin{abstract}
Levy's theorem 'A second order parallel symmetric non-singular tensor in a real space form is proportional to the metric tensor has been generalized by showing that it holds even if one assumes the second order tensor to be parallel (not necessarily symmetric and non-singular) in a real space form of dimension greater than two. Analogous result has been established for a complex space form. It has been shown that an affine Killing vector field in a non-flat complex space form is Killing and arıalytic.
\end{abstract}

KEY WORDS AND PHRASES. Second order parallel tensor, Real space form, Complex space form, Affine Killing vector field, Analytic vector fiéld.

1980 AMS SUBJECT CLASSIFICATION CODE. 53C, 55.

\section{INTRODUCTION.}

In 1923, Eisenhart [1] proved that if a positive definite Riemannian manifold admits a second order parallel symmetric tensor other than a constant multiple of the metric tensor, then it is reducible. In 1926, Levy [2] proved that a second order parallel symmetric non-singular (with non-vanishing determinant) tensor in a space of constant curvature is proportional to the metric tensor. The purpose of this paper is to present a generalization over Levy's theorem for dimension greater than two in the form of Theorem 1 and its analogue in a Kaehlerian manifold of constant holomorphic sectional curvature (also called a complex space form) in the form of Theorem 2. Using Theorem 2, it has been proved in Theorem 3 that an affine Killing vector field in a non-flat complex space form is Killing and analytic.

Let $M$ denote an n-dimensional pseudo-Riemannian manifold with its metric tensor $g$ of arbitrary signature and Levi-Civita connection $\nabla$. Let $R$ denote the Riemann curvature tensor of $M$. If $h$ is $a(0,2)$-tensor which is parallel with respect to $\nabla$ then we can show easily that

$$
h(R(X, Y) Z, W)+h(Z, R(X, Y) W)=0
$$




\section{A GENERAI.IZ.ATION OF LEVY'S 'IHFOREM.}

Wo present the following gencralization over levy's theorem:

THEOREM 1. A second order parallel tensor in a non-flat real space form of dimension $\mathrm{n}>2$ is proportional to the metric lensor.

PROOF: For a real space form $M$ with constant sectional curvature $k$, we have

$$
H(X, Y) Z=k\{g(Y, Z) X-g(X, Z) Y\}
$$

Note that $k \neq 0$, by hypothesis. Use of (2.1) in (1.1) gives

$$
g(Y, Z) h(X, W)-g(X, Z) h(Y, W)+g(Y, W) h(Z, X) \cdot g(X, W) h(Z, Y)-0
$$

Contraction at $X$ and $W$ with respect to an orthonormal frame in $M$, provides

$$
(\operatorname{tr} . H) g(Y, Z)-h(Y, Z)+h(Z, Y) \cdot \mathbf{n h}(Z, Y)-0
$$

where $H$ is a $(1,1)$-tensor metrically equivalent to h. Anti-symmetrization of (2.3) shows that $h$ is symmetric. Fventually (2.3) reduces to

$$
h=\frac{\operatorname{lr} \cdot H}{n} g
$$

Now, tr.H is constant, as $H$ is parallel. Hence (2.4) proves the theorem.

REMARK 1. That the theorein 1 does not hold for $n=2$, can be seen by considering the 2-sphere $S^{2}$. It is known that $S^{2}$ carries a Kaehlerian structure (see the beginning of section 3 ) whose Kaehlerian 2-form is a parallel tensor.

\section{ANALOGUF OF THEORFM 1 FOR A COMPILX SPACE FORM.}

Before presenting an analogue of Theorem 1 for a complex space-form, we would like to recall the basic structure of a complex space form $M(c)$. $M(c)$ is a Kaehlerian manifold of constant holomorphic sectional curvature $c$, with its complex structure tensor $\mathrm{J}: \mathrm{J}^{2}=-\mathrm{I}$, Kaehlerian metric: $\mathrm{g}: \mathrm{g}(\mathrm{JX}, \mathrm{JY})=\mathrm{g}(\mathrm{X}, \mathrm{Y})$, Kaehlerian 2-form $\Omega: \Omega(X, Y)=g(X, J Y)$ and the Kaehlerian connection $\nabla: \nabla J=0$.

THнORFM 2. A second order parallel tensor in a non-flat complex space form is a linear combination (with constant coefficients) of the underlying Kaehlerian metric and Kuehlerian 2-form.

PROOF: For a complex space form $M(c)$, it is known [3] that

$$
R(X, Y) Z=\frac{c}{4}[g(Y, Z) X-g(X, Z) Y+g(J Y, Z) J X-g(J X, Z) J Y+2 g(X, J Y) J Z]
$$

Plugging the value of $H$ from (3.1) into (1.1) and contracting at $X$ and $w$, provide

$$
\begin{aligned}
(\operatorname{tr} . H) g(Y, Z) \cdots h(Y, Z) & \cdot(\mathbf{n}+2) h(Z, Y)+\operatorname{tr} \cdot(H J) g(J Y, Z) \\
+ & g(H J Y, J Z)+2 g(H J Z, J Y)=0
\end{aligned}
$$

Symmetrization and anti-symmetrization of (3.2) yield: 


$$
\begin{aligned}
& (\mathbf{n}+3) h_{\mathbf{s}}(\mathrm{Y}, Z)-3 \mathbf{h}_{\mathbf{s}}(\mathrm{JY}, \mathrm{JZ})=(\mathrm{tr} . \mathrm{H}) \mathrm{g}(\mathrm{Y}, \mathrm{Z}) \\
& (n+1) h_{a}(Y, Z) \cdots h_{a}(J Y, J Z)=(t r . H J) g(Y, J Z)
\end{aligned}
$$

Replacing $Y, Z$ by $J Y, J \%$ respectively in (3.3) and subtracting the resultant equation from (3.3), provide the relation:

$$
h_{s} \frac{t r \cdot H}{n} g
$$

Likewise; replacing $Y, Z$ by $J Y, J Z$ respectively in (3.4) and eliminating $h_{a}(J Y, J Z)$ from the resultant equation and (3.4), provide the relation:

$$
h_{a}=\frac{\operatorname{tr} \cdot(H J)}{n} \Omega
$$

By summing up (3.5) and (3.6) we obtain the expression:

$$
h=\frac{1}{n}[(\operatorname{tr} . H) g+(\operatorname{tr} . H J) \Omega]
$$

Now as both $H$ and $J$ are parallel with respect to $\nabla$; therefore tr.H and tr.HJ are constants. Thus, Equation (3.7) proves the theorem.

COROLLARY. The only symmetric (anti-symmetric) parallel tensor of type $(0,2)$ in a non-flat complex space form is the Kaehlerian metric (the Kaehlerian 2-form) up to $n$ constant multiple.

REMARK 2. The anti-symmetric case of the above corollary agrees well with the following result [3]: 'In a compact Kaehlerian space of constant holomorphic sectional curvature $c>0$, we have $B_{2 l}=1, B_{2 l+1}=0$ for $\left.0 \leqslant 2 l, 2 l+1 \leqslant n\right)^{\prime}$. Taking $l=1$, the second Betti number $B_{2}=1$ for a compact $M(c)$ with $c>0$. Thus the only harmonic 2-form in such a space is the Kaehlerian 2-form $\Omega$ (Note that $\nabla \Omega=0$ implies $d \Omega=0$ and $\delta \Omega=0$, that is, $\Omega$ is harmonic).

THEOREM 3. An affine Killing vector field in a non-flat complex space form is Killing and analytic.

PROOF: If $\xi$ is an affine Killing vector field in a non-flat $M(c)$, then the Lie-derivative $L \xi g$ of the metric tensor $g$ is a second order parallel tensor. A direct application of the symmetric case of the corollary to Theorem 2, shows that $L_{\xi g}=a g$ (a being a constant). The last equation implies that $L_{\xi} R i c=0$ (Ric denotes the Ricci tensor of $M(c))$. Now, we know [3] that $M(c)$ is an Finstein space, that is, $R i c=\frac{n+2}{4} \mathrm{cg}$. Taking the Lie-derivatives of both sides along $\xi$ and noting $c \neq 0$, obtain $L_{\xi} g-0$. Hence $\xi$ is Killing. To prove the remaining part, we first observe the identity [4]:

$$
\left(\mathrm{I}_{\xi} \nabla_{\mathrm{X}} \mathrm{J}-\nabla_{\mathrm{X}} \mathrm{L}_{\xi} \mathrm{J}-\nabla_{[\xi, X]} \mathrm{J}\right) \mathrm{Y}=\left(\mathrm{L}_{\xi} \nabla\right)(\mathrm{X}, \mathrm{JY})-\mathrm{J}\left(\left(\mathrm{L}_{\xi} \nabla\right)(\mathrm{X}, \mathrm{Y})\right)
$$

But $\nabla_{X} J=0$ and $L_{\xi} \nabla=0$ and therefore the above identity implies

$$
\nabla_{X} L_{\xi} J-0
$$


As $\xi$ is Killing, it follows from the relation: $\Omega(Y, Z)=g(J Y, Z)$, that

$$
\left(\nabla_{X} I_{\xi} \Omega\right)(Y, Z)=g\left(Y,\left(\nabla_{X} I_{\xi} J\right) Z\right)
$$

The last equation; together with (3.8), yields

$$
\nabla_{X^{L}} L \Omega=0
$$

Note that $\Omega$ is anti-symmetric and therefore, so is $L \xi \Omega$. In view of (3.9) and the anti-symmetric case of the corollary to Theorem 2 , we obtain $L \xi \Omega=b \Omega$ (b is constant). Using the above relation we derive

$$
\mathbf{L}_{\xi} \mathrm{J}=\mathbf{b J}
$$

Now, $\quad L_{\xi}\left(J^{2} Y\right)=\left(L_{\xi} J\right)(J Y)+J\left(\left(L_{\xi} J\right) Y\right)+J^{2}\left(L_{\xi} Y\right)$ shows that

$$
\left(\mathrm{L}_{\xi} \mathrm{J}\right)(\mathrm{JY})+\mathrm{J}\left(\left(\mathrm{I}_{\xi} \mathrm{J}\right) \mathrm{Y}\right)=\mathbf{0}
$$

Use of (3.10) and (3.11) readily gives $b=0$. Consequently (3.10) reduces to $L_{\xi} J=$ 0 . Hence $\xi$ is an analytic vector field [3]. This completes the proof.

REMARK 3. In Theorem 3 we have proved that a Killing vector field in a non-flat complex space form is analytic vector field of $J$. One can compare this result with the following result of Yano [3]: 'A Killing vector field in a compact Kaehler space is analytic'. Our result assumes the vector field to be just affine Killing and proves it to be Killing and analytic in a complex space form (not necessarily compact), whereas Yano's result proves a Killing vector to be analytic if the space is compact Kaehler (not necessarily of constant holomorphic sectional curvature).

ACKNOWLEDGEMENT. The author expresses his sincere thanks to the Lord Sri Satya Sai Baba, for His grace through which this work has been done. The author is thankful to Professor David E. Blair for pointing out that theorem 1 is invalid for dimension 2, in view of Remark 1.

\section{REFERENCES}

1. L.P. Eisenhart, Symmetric tensors of the second order whose first covariant derivatives are zero, Trans. Amer. Math. Soc. 25 (1923), 297-306.

2. H. Levy, Symmetric tensors of the second order whose covariant derivatives vanish, Annals of Maths. 27 (1926), 91-98.

3. K. Yano, Differential geometry on complex and almost complex spaces, Pergamon Press, New York, 1965.

4. K. Yano, Integral formulas in Riemannian geometry, Marcel Dekker, Inc.., New York, 1970. 


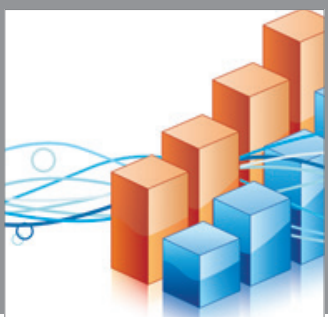

Advances in

Operations Research

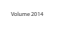

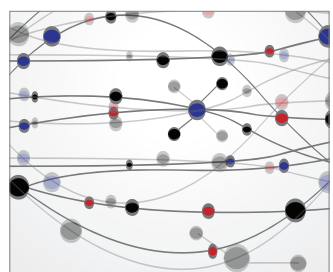

\section{The Scientific} World Journal
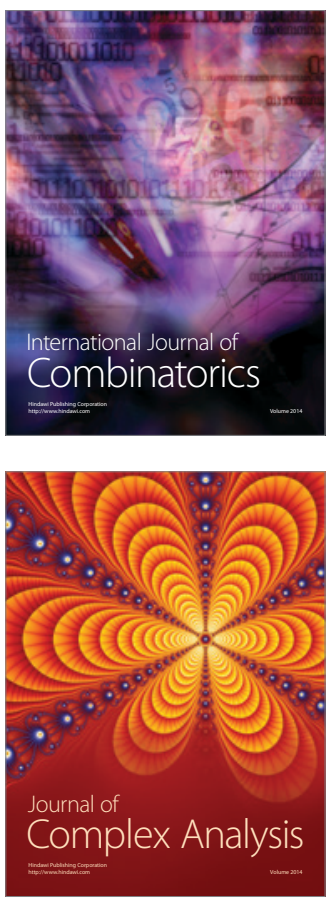

International Journal of

Mathematics and

Mathematical

Sciences
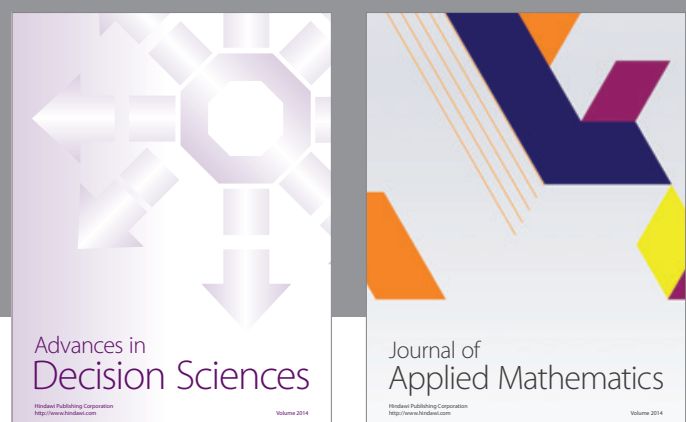

Journal of

Applied Mathematics
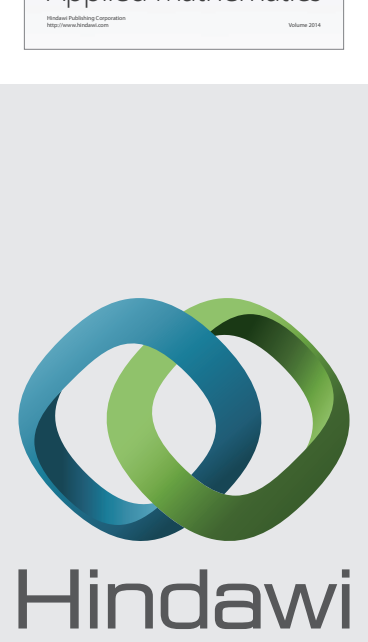

Submit your manuscripts at http://www.hindawi.com
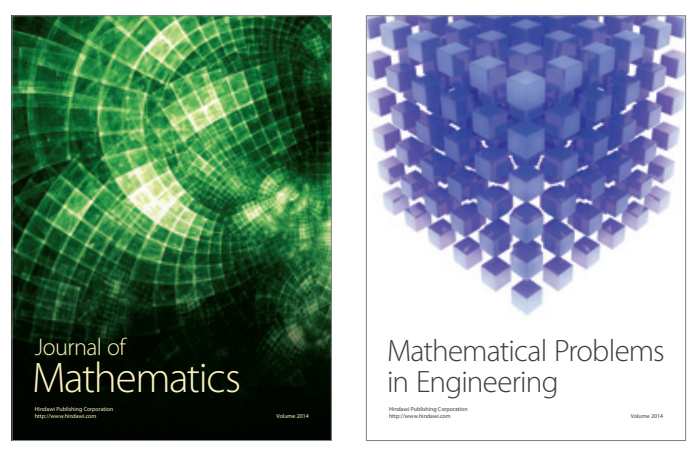

Mathematical Problems in Engineering
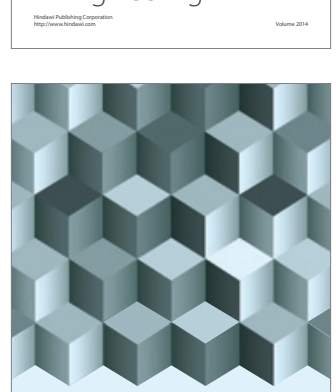

Journal of

Function Spaces
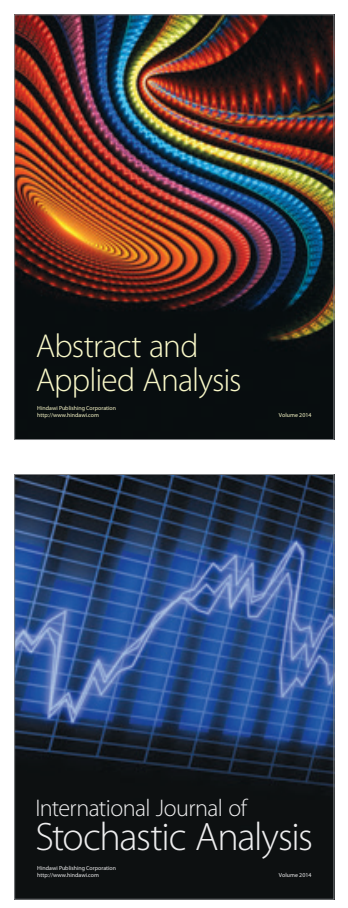

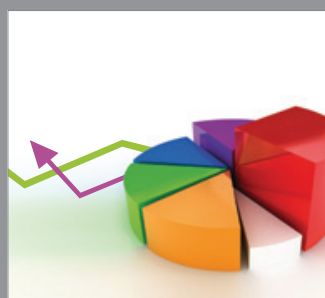

ournal of

Probability and Statistics

Promensencen
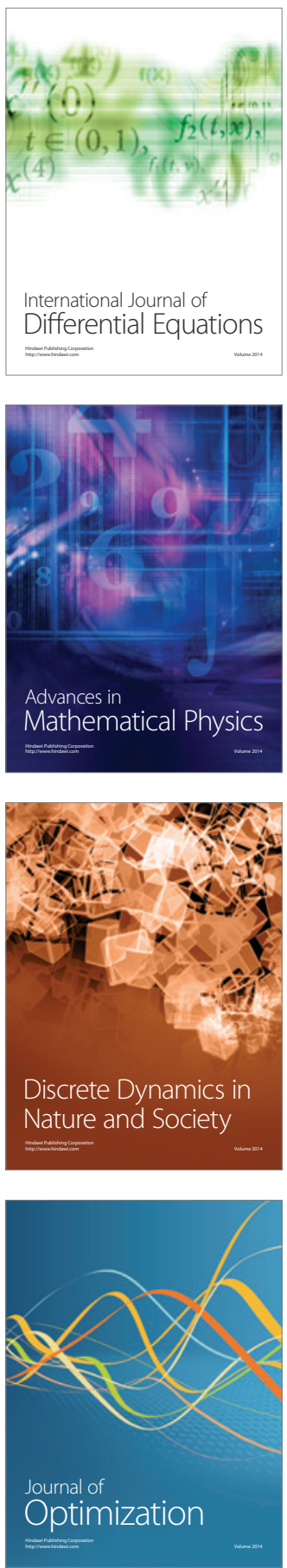